\title{
REVIEW
}

\section{Evolution of Acinetobacter baumannii infections and antimicrobial resistance. A review}

\author{
Sonia Elena Popovici ${ }^{1}$, Ovidiu Horea Bedreag ${ }^{2}$, Dorel Sandesc ${ }^{2}$ \\ 1"Pius Branzeu" Emergency County Hospital, Timisoara, Romania \\ 2 Faculty of Medicine, "Victor Babes" Univeristy of Medicine and Pharmacy, Timisoara, Romania \\ Correspondence to: \\ Sonia Elena Popovici, MD \\ Clinic of Anesthesia and Intensive Care "Pius Branzeu" Emergency County Hospital, Timisoara, Romania, \\ Bulevardul Liviu Rebreanu, Nr. 156, Cod 300723, Timișoara \\ E-mail: popovici.sonia@yahoo.com
}

\section{Conflicts of interests}

Nothing to declare

\section{Acknowledgment}

None

Funding: This research did not receive any specific grant from funding agencies in the public, commercial or not-for profit sectors.

Keywords: Acinetobacter baumannii, hospital-acquired, antimicrobial resistance.

These authors take responsibility for all aspects of the reliability and freedom from bias of the data presented and their discussed interpretation.

Central Eur J Clin Res 2019;2(1):28-36

Received: 12.12.2018, Accepted: 15.01.2019, Published: 25.03.2019

Copyright $\odot 2018$ Central European Journal of Clinical Research. This is an open-access article distributed under the Creative Commons Attribution License, which permits unrestricted use, distribution, and reproduction in any medium, provided the original work is properly cited.

\section{Abstract}

The emergence of multi-drug resistant Acinetobacter spp involved in hospital-acquired infections, once considered an easily treatable pathogen, is troublesome and an immense burden for the modern medical systems worldwide. In the last 20 years the medical community recorded an increase in the incidence and severity of these infections as therapeutic means tend to be less and less effective on these strains. The ability of these bacteria to rapidly develop resistance to antimicrobial agents by continuously changing and adapting their mechanisms, their ability to survive for long periods of time in the hospital environment and the multitude of transmission possibilities raises serious issues regarding the management of these complex infections. The future lies in developing new and targeted methods for the early diagnosis of $A$. baumannii, as well as in the judicious use of antimicrobial drugs. This review details the evolution of the pathogenicity of this microorganism, together with the changes that appeared in resistance mechanisms and the advancements in molecular testing for the early detection of infection.

\section{Introduction}

The critically ill patient as one of the biggest challenges in the medical world has a con- 
stant need of complex management which also involves the prevention and treatment of associated infections. The length of stay in the ICU, the mechanical ventilation time, the invasive techniques used, as well as the nutrition strategy all make the critically ill patient susceptible to infection and influence the clinical outcome and mortality rates. It was proven that the mortality risk is 2.48 times higher in critically ill patients with hospital-acquired infections especially in the case of patients with respiratory disease [1].

Bacterial infections are a heavy burden on the medical systems and on patients worldwide, with the most threatening being pathogens of the respiratory tract, enteric pathogens and HIV-associated infections. Finding new treatment options represents a constant challenge for the medical community, as the fight is against the continuously evolving mechanisms of microbial resistance. New hope comes from the recent advances in the field of genetics, mainly DNA sequencing that offer the possibility of comparing the genomes of the same strains over a period of time in order to identify genetic changes signifying the development of adaptation techniques of the microorganism to certain drugs or to the environment [2]. Along the years research groups have struggled to elucidate the resistance mechanisms of certain pathogens involved in acute infections, such as Neisseria meningitidis, Pseudomonas aeruginosa, and Acinetobacter baumannii, with a soft focus on their genomic evolution.

\section{Background}

The alarming increase in the incidence of infections determined by opportunistic microorganisms, especially as epidemiologic events such as nosocomial infections, have determined researchers in the fields of microbiology and molecular biology to carry out studies on these certain types of pathogens. Acinetobacter baumannii is an ubiquitary bacterium that colonizes over $40 \%$ of healthy adults that come into contact, with a higher incidence seen in the medical personnel.

Belonging to a wider class called Gammaproteobacteriae, germs in the Acinetobacter genus have long been considered as opportunists, usually involved in less severe infections. Genus Acinetobacter as we nowadays call it was first discovered in 1911 by Martinus Beigerinck, as a gram-negative bacterium [3]. Only in 1970 was this pathogen recognized as being significantly involved in hospital infections, but with a high sensitivity to common antimicrobials used at the time. However, in the last 30 years an increased incidence and severity of the in- fections have been reported, especially in the case of hospital-acquired infections, raising a red flag related to the lack of therapeutic means for the ever-evolving multidrug-resistant strains of this bacterium. Together with technological advancements, in 1986 four new genomic species were proposed after using DNA-DNA hybridization: 3TU, 13 TU, Acinetobacter baumannii, and Acinetobacter calcoaceticus which together form the " $A$. baumannii complex" [4], as they share common properties that make them hard to distinguish from each other. A. baumannii infections associated with medical interventions were proven to have high-virulence which altered the belief that it was a low-virulence pathogen acting as an opportunist [5]. Their potential of rapidly developing resistance to antimicrobial agents, their capacity of surviving in the hospital environment for long periods of time, and the numerous transmission mechanisms have raised serious issues worldwide regarding the treatment and management of hospital-acquired Acinetobacter spp. infections [6]. Wright et al. carried out a genomic analysis of $A$. baumannii isolated from the same patient during their hospital stay and have proven that antibiotic treatment and host response led to enrichment in mutation [7].

\section{Microbiology - current classification and relevance in the clinical setting}

The genus Acinetobacter encompasses opportunistic pathogens, gram-negative coco-bacilli in pairs, with dimensions between 1 and $1.5 \mu \mathrm{m}$ that do not present mobility, and produce a catalase enzyme (catalase-positive) but does not produce cytochrome oxidase (oxidase-negative) or urease. Regarding nutritional mechanisms these pathogens are aerobic, non-fermenting, and non-fastidious [8]. More recent classification strategies have proposed to classify the genus Acinetobacter as part of the Moraxellaceae family and of the more extensive Gammaproteobacteria class.

The $A$. baumannii complex includes the three most clinically important species that have been involved in community-acquired and hospital-acquired infections in humans, as well as a fourth specie, A. calcoaceticus, frequently found in water and soil that does not inflict diseases in the human population. Apart from the "Acinetobacter complex" Bouvet and Grimont, and later Bouvet and Jeanjean [4], described further genospecies through DNA-hybridization including: A. haemolyticus, A. johnosnii, A. junii, A. Iwoffii, and $A$. radioresistens. In the last two decades additional species have been identified, but out of the total 10 only 3 are of human origin: 
A. parvus, $A$. schindleri, and A.ursingii $[9,10,11]$. Up until now the following species of the Acinetobacter genus have been identified, with their type and reference strains mentioned in the brackets: A. baumannii (ATCC19606T) - with two genomic species, A.baylyi (DSM14961T), A. bouvetii (DSM 14961T), A. calcoaceticus (ATCC 23055T) - one genomic specie, A. gerneri (DSM 14967T), A. grimontii (DSM 14968T), A. haemolyticus (ATCC 17906T) with 4 genomic species, A. johnsonii (ATCC 17909T) with 7 genomic species, $A$. junii (ATCC 17908T) with 5 genomic species, A. lowffii (ACTC 15309, ATCC 9957) with 8, respectively 9 genomic species, A. parvus (NPH384T), A. radioresistens (IAM 13186T) with 12 genomic species, A. schindleri (NIPH1034T), A. tandoii (DSM 14970T), A. tjernbergiae (DSM 14971T), A. twoneri (DSM 14962T), A. ursingii (NIPH137T), A. venetianus (ATCC 31012, ATCC 19004, 10090 - close to 13TU) $[4,9,12,13,14]$

\section{Identification of Acinetobacter strains}

For the identification of Acinetobacter strains one must use a combination of phenotypic and genotypic tests that must be characterized by high reproducibility, good discrimination strength, and that are easily carried out and easy to interpret [15]. Samples in the case of ICU patients can be collected from different sites such as: lower respiratory tract secretions, urine, wound secretion, central lines, or even skin. Along the years this species has become more and more aggressive, as well as more resistant to even the most modern therapies. There are different methods for testing but unfortunately standard techniques used in the laboratory will only identify the genus and not the species [14]. Phenotypic identification methods such as the biochemical profile, phagocytic typology, surface antigens profile, antibiotic susceptibility etc. represent the expression of certain genetic structures, and therefore are susceptible to variability due to the influence of the culture environment, bacterial growth phase, spontaneous mutations [14]. Among the most used biotyping methods one of the most widely used in the clinical setting is the API 20NE and API 20E (BioMerieux, France) test. Complementary growth at $44{ }^{\circ} \mathrm{C}$ is sometimes required although the test has high reliability [15].

\section{Molecular techniques}

Genetic methods analyze the microorganism's genetic structure and include the polymorphism of the enzymatic restriction patterns of the total DNA, as well as the presence or ab- sence of specific structures in the chromosomal or extra-chromosomal DNA sequence. Natural variability does not influence these methods as much but they can be affected by insertion, deletions, and point mutations. Fortunately the incidence of these defects is lower, and in many cases they can represent diagnostic or epidemiologic biomarkers.

A. baumannii species present indigenous plasmids and therefore plasmid analysis is one method that has been used for the epidemiological typing but only after taking into account that plasmids can be gained or lost in the transformation process [16].

Ribotyping, although labor-intensive and with limited discriminatory power, has been used to identify species of Acinetobacter. The method applied in the past in epidemiologic studies uses EcoRI, Clal, and Sall in order to obtain the restriction of DNA; after this step electrophoresis must be carried out, followed by blotting and hybridization with a probe derived from E.Coli ribosomal RNA [9]. Because the technique is both expensive and time-consuming, and requires specific equipment it was replaced by pulse-field gel electrophoresis (PFGE).

Pulse-field gel electrophoresis (PFGE) remains the gold standard for identifying Acinetobacter baumannii [9]. It is a so-called comparative typing system as it uses Apal to impose restriction on chromosomal DNA [17], followed by electrophoresis for the separation of these fragments and by the comparison of the fingerprint profiles. Reproducibility between different laboratories is also possible which would help in the detection of epidemic strains in a certain hospital or even nation-wide [18].

Applied fragment length polymorphism analysis (AFLP analysis) is a semiautomated procedure and a powerful tool in determining taxonomy in bacterial strains, but being a method that requires high level equipment and standardization it is found in reference laboratories and it is not used in the daily practice for routine analysis of $A$. baumannii [19].

The sequence analysis for $16 \mathrm{~S}$ rDNA genes and $r p o B$ genes are molecular identification methods that are widely accepted, as well as the PCR-RFLP molecular technique. PCR Amplification of bla ${ }_{\text {OxA-51 }}$ and CHROMagar Acinetobacter/MDR Technique are other tests that together with the antimicrobial susceptibility testing can aid in a correct and goal directed therapy for $A$. baumannii infections [20].DNA extraction is another option, as well as PCR reactions.

As all gram-negative bacteria, the Acinetobacter species expresses lipopolysac- 
charides (LPSs), present on the outer surface of the microorganism [21], that were shown to be of smooth phenotype after serological and chemical analysis [22-24]. Monoclonal antibodies (MAbs) against the LPSs of the $A$. baumannii strains were generated; MAb S48-3-13 and MAb S48-3-17 were found to react with certain strains [12]. Another pair of mouse monoclonal antibodies used against $A$. baumannii ATCC 19606 was reported by Baig et al [25], mainly F241G3sc2 and F241G6sc2 that have proven to be highly sensitive and demonstrated applicability in the clinical practice by use of ELISA testing and Western Blot analysis, and therefore can be used as rapid detection tools [25]. Moreover, in the last years, humanized MAbs have emerged as possible effective treatment options or prevention options for $A$. baumannii infections, especially with multi-drug resistant (MDR) or extensive-drug resistant (XDR) strains.

Another very important part is determining the sensitivity/resistance profile to antimicrobial drugs. This test can be applied to all strains and is easy to perform and interpret. The laboratory clinician will determine either the inhibition area, or the minimum inhibitory concentration (MIC). The resistance profile is very variable and can rapidly change in the same patient or in the same epidemiologic outburst. Another example of molecular and biochemical methods that are useful in the detection of antimicrobial resistance profiles is the detection of carbapenemase-producing Acinetobacter baumannii, as these strains have developed and evolved on a fast-raging pace over the last years. Two of these methods were described by Thoma et. al, CarbaAcinetoNP and BlueCarba Test, together with PCR testing for carbapenemases [26]. Testing the ability of producing large-spectrum betalactamases (BLSE) can also be achieved, through the double-disc method. Genotyping methods for MDR $A$. baumannii include: amplified fragment length polymorphism analysis (AFLP), PFGE, multiple locus variable number tandem repeat analysis (MLVA), single locus genotyping, resistance island typing, and next-generation whole-genome sequencing (NG WGS) with each having different genetic targets and specific features.

\section{Pathogenicity and pathogenesis of Acinetobacter spp}

The species of the Acinetobacter genre can be involved in hospital-acquired infections, the most commonly identified in the clinical setting being $A$. baumannii, more specifically the genospecies included in the "Acinetobacter baumannii complex", that cannot be distin- guished biochemically [27]. This pathogen is able to infect and survive on hospital surfaces for long periods of time and therefore lead to hospital-acquired infections in susceptible patients and nosocomial outbreaks $[8,28]$. The vast majority of clinically relevant infections appear in hosts with a compromised immune system, where the virulence factors allow colonization and infection. Among these virulence factors we mention: production and release of exopolysaccharides, the LPS that can contribute to its pathogenicity through their mutagenic properties and their ability to induce TNF-alpha synthesis [29]. Mortality rates in hospitalized patients infected with this pathogen reach $26 \%$, while mortality in the ICU patient group reaches 43\% [30].

Acinetobacter baumannii has been isolated from all culture sites, but the most common infection and colonization site remains the inferior respiratory tract. It is the main pathogen incriminated in ventilator-associated pneumonia (VAP) but lately it has been proven that it can also lead to community-acquired pneumonia in patients that present with further risk factors such as COPD, alcoholism, heavy smoking, and diabetes, with a high prevalence in Asia as well as Australia [31]. Among the risk factors for developing an infection with $A$. baumannii Arodino et al. [32] mentioned bronchoscopy and oxygen therapy as invasive procedure performed outside the ICU or before the admission in the ICU, together with obesity or malnutrition, severe liver disease, and the type and duration of antibiotic therapy. Apart from respiratory infections cause by $A$. baumannii, other types and localizations of infections are also possible such as meningitis, bloodstream and central-line associated, skin or wound.

A. baumannii also has a horizontal transmission pattern, from one patient to the other when they are hospitalized in close vicinity and it is also capable of colonizing surfaces which apart from hospital furniture can also refer to feeding tubes. Wrong manipulation and lack of hygiene will therefore lead to the spread of the infection. In the ICU wards the main source for the infection are catheters of all types, equipment used for mechanical ventilation and other type of ventilatory support, and suction devices [9].

The pulmonary colonization and infection begins through the adherence to host cells that can be followed by the formation of a biofilm, dependent on the existence of pilli on the bacterial outer membrane. A. baumannii forms biofilms on both biotic and abiotic surfaces which makes the microorganism metabolically inert. It is because of this mechanism that vir- 
ulence is increased as antibiotics cannot penetrate or act on metabolically inert organisms [8]. Biofilm production is deeply influenced by other virulence factors such as the family of BAP proteins (biofilm associated protein), OmpA, and BAP-like proteins [33].

Ab0mpA (outer membrane protein) is a major membrane protein that is highly immunogenic and leads to an increase expression of cytochrome $\mathrm{c}$ which is a proapoptotic molecule. High concentrations of AbOmpA induce apoptosis of dendritic and epithelial cells that leads to the destruction of the mucosa and allows the bacteria to penetrate deeper tissues [34]. In low concentrations Ab0mpA stimulates the expression of CD80, CD86, CD40, Major Histocompatibility Complex (MHC) class I, and MHC class II, as well as the production of interleukin 1 (IL1); it also determines epithelial cells to become more responsive to ligands by increasing the surface expression of Toll-Like Receptor 2(TLR 2) which plays an important role in the pulmonary host defense mechanism. The membrane LPS of $A$. baumannii has been proven to be involved in the pathogenesis of sepsis by inducing pro-inflammatory cytokine synthesis and by binding to CD14 and the TLR4/MD-2 complex [35].

Neutrophil depression significantly reduces the cytokine levels showing that these cells play a crucial role in the pro-inflammatory response of the lung. Recruiting a high number of neutrophils in the upper and lower airway and in the lung parenchyma was correlated with the eradication of $A$. baumannii infection in the lungs [36]. Macrophages on the other hand are the first defense line and involved in the phagocytosis in the lower airways. They express a variety of cellular recognition molecules and studies have proven that they have a direct role in the local defense against $A$. baumannii through a NO-dependent mechanism that need an intact and functional microfilament and microtubule system in order to function adequately [37]. Understanding the virulence mechanisms of $A$. baumannii can facilitate the development of new immunotherapies, as well as new antibiotics.

\section{Mechanisms of antimicrobial resistance and their evolution pattern}

From once being only an opportunistic bacterium, easy to treat even with narrow-spectrum antibiotics $A$. baumannii has evolved to being capable of mutations associated both with antimicrobial therapies and with the host response. Since 1928, with the great discovery of Alexander Fleming - Penicillin - people have thought that they discovered a "magic weapon" that would eradicate infections from the face of the Earth, not knowing the capability of bacteria of counteracting this fight. The first penicillin-resistant microorganisms have been reported only one year after the first administration of penicillin, and methicillin soon followed the same pattern. With regard to $A$. baumannii up until 1970 infections were easily treatable but high rates of resistance emerged as early as 1975. In the late years many valuable drugs are no longer efficient in the treatment of these infections, including cephalosporins, tetracycline, most aminoglycosides and cefoxitin $[9,38]$. The current therapeutic regimens include carbapenems, although recent studies have shown increased resistance, fluroquinolones, and sometimes broad-spectrum chephalosporines if the microorganism is susceptible. Even in susceptible strains in the late years MICs have increased [39]. Based on their research Marvig et al., concluded that the population of $A$. baumannii includes at least nine distinct clonal lineages worldwide [40].

In their study, Hua et al. [41] carried out the analysis of 172 genomes of isolated $A$. baumannii, from 86 different patients to research the evolution of the microorganism within its host and they have shown that worldwide $A$. baumannii comprises of three major international lineages: EC I, EC II, and EC III. Out of the three the dominant one is EC II with its subgroup ICL-II that represents the carbapenem-resistant A. baumannii. After analyzing the single nucleotide polymorphism (SNPs) for within-patient diversity they have shown that over 15 genes has been mutated more than one time. A gene with a significant role in capsule synthesis, ptk has been shown to be mutated the most, with the mutant microorganism presenting higher motility levels. Another mutation was that of adeRS that increased the resistance to tigecycline. Hua et al have also shown that $A$. baumannii is resistant to major antimicrobial drugs and that the resistance increases together with its evolution within the host [41].

Since the 1980s the spread of $A$. baumannii infections has been a problem across Europe, with outbreaks in hospitals in western European countries such as Germany, the Netherlands, France and Italy [42]. After identifying the epidemic strains infecting these hospitals it was proven that transmission is also possible between hospitals, most probably via transfers of infected or colonized patients [43]. The possibility of spread of multi-drug resistance $A$. baumannii is possible not only from a patient to the other or from one hospital to the other, but also at a national and even international level, a fact that was proven by many different stud- 
ies. Turton et al have described the multi-hospital epidemic given by the "Southeast clone" and by the Oxa-23, Oxa-1, and Oxa-2 clones (all MDR-A. baumannii) which infected patients in English hospitals and disseminated through Southeast England [44]. Another example is the epidemic that affected northern and southeastern France, where a VEB-1 ESBL A. baumannii clone spread in a total of 55 medical centers [45]. Moreover, hospital outbreaks in Northern Europe, Eastern Europe and other EU countries such as Greece and Spain were reported with three international clones of $A$. baumannii, mainly the European clone I, II, and III. Epidemiologists and researchers could not find the link between the outbreaks given by these European clones [46].

The resistance mechanisms to Cephalosporins in the case of $A$. baumannii is given by the natural production of Acinetobacter-derived cephalosporinase (also called AmpC $\beta$-lactamase or, shorter, $A D C)$. In this case the production of ADC cannot be repressed, and what makes it even more dangerous is the fact that it can even be enhanced by the insertion of certain sequences in the gene which in the end leads to a high level of resistance $[47,48]$. Certain strains of $A$. baumannii also have the capacity of producing, apart from ADC, the extended-spectrum $\beta$-lactamase (ESBL) which also renders them resistant to cephalosporins [49].

On the other hand, the production of carbapenemases is the most important mechanism for resistance of $A$. baumannii to carbapenems. Carbapenemases can be produced by the microorganism naturally, such as the group of OXA-51 chromosomally encoded carbapenemases, but they can also be acquired. In the same manner as for the ADS, resistance can be reinforced through an insertion sequence upstream of OXA-51 gene which will lead to increased MICs for carbapenems [9,50]. In the recent years $A$. baumannii has acquired carbapenemases in the non-OXA group, that up until recently were specific for the Enterobacteriaceae.

Sulbactam, which used to be a solution for the $\beta$-lactamase producing group, is given as a combination with either ampicillin or cefaperazone, but it also has its own activity against A. baumannii. However, in the last decades through the production of TEM-1 $\beta$-lactamase and through low expression of penicillin-binding pretin (PBP2), A. baumannii has managed to develop resistance to this drug $[51,9]$.

The activity of aminoglycosides is affected by the ability of the bacteria to produce a variety of aminoglycoside-modifying enzymes
[52]. This class of antimicrobials exerts their activity through the inhibition of protein synthesis. This is achieved by binding to the ribosomal RNA (16S) of the ribosomal subunit (30S) but $A$. baumannii is capable of producing ArmA which is a $16 \mathrm{~S}$ ribosomal RNA methyltransferase [9], protecting the aminoglycoside-binding site from binding the drug [53].

Colistin is an antimicrobial with bactericidal activity and exerts its effect by binding to lipid $A$, an endotoxin of gram-negative bacteria, and the interior layer of the LPS. Modification of lipid A lead to resistance to colistin, by the addition of phosphoetanolamine [56]. Another mechanism that renders colisitin ineffective is the total loos of the LPS [57].

A. baumannii also presents both moderate and high-level resistance to fluorochinolones. As a mechanism of action they bind to the topoisomerase IV and DNA gyrase [9], therefore disrupting the synthesis of DNA and inducing cell death. The microorganism substitutes amino acids in the genes that encode target proteins for the quinolone resistance determining region [54], this leading to high levels of resistance to fluorochinolones. Moderate levels of resistance are achieved by the overexpression of active efflux pumps $[55,9]$.

\section{Conclusions}

The emergence of multi-drug resistant Acinetobacter spp involved in hospital-acquired infections is troublesome and an immense burden for the modern medical systems worldwide, especially because of the ever-developing strains capable of ESBL and carbapenemases production that led to pan-resistance (extensive resistance to carbapenems, aztreonam, all cephalosporins, aminoglycosides, and fluorochinolones). These strains have a high potential of survival for long periods of time in the hospital environment, leading to contamination of medical equipment and prosthetic devices.

The risk factors for hospital-acquired infections with multi-drug resistant $A$. baumannii include admission in the intensive care unit, mechanical ventilation, invasive procedure and surgical interventions, using prosthetic devices, as well as previous antibiotic treatment with carbapenems, $3^{\text {rd }}$ generation cephalosporins, and quinolones.

Modern testing for $A$. baumannii strains and for the resistance profiles should be available to all healthcare authorities. However, genetic testing is still not accessible everywhere, but with future reduction of costs for the DNA library preparation could make genomics a tool that could be used for routine surveillance [2]. 
As a future perspective population genomics will help researchers and clinicians altogether to better understand both the exact etiology but also the epidemiology and evolving possibilities of infections caused by $A$. baumannii.

Therapeutic options are limited as the microorganism continues to evolve and to develop new and more complex resistance mechanisms to modern antimicrobials. Hospital-acquired infections with $A$. baumannii lead to a worse clinical outcome of the patients, increasing the financial burden related to medical costs because of longer stays in the ICU and on other hospital wards and complex antimicrobial treatments. Passive and active surveillance as well as prevention and control methods could reduce the rate of $A$. baumannii infections. Therefore surveillance of the antimicrobial therapy in each hospital, with guidelines respecting the resistance profiles, and constant training of all medical personnel regarding prevention methods would have a favorable impact on the issue.

\section{References}

1. Bueno-Cvanillas A, Delgado-Rodriguez $M$, et al. Influence of nosocomial infection on mortality rate in an intensive care unit. Crit Care Med 1994; 22(1):55-60

2. Wilson DJ. Insights from genomics into bacterial pathogen populations. PLoS Pathog. 2012;8(9):e1002874.

3. Nemec A, Musilek M, Mxinerova $M$, etl al. Acinetobacter beijerinckii $s p$ nov and Acinetobacter gyllenbergii sp nov., haemolytic organisms isolated from humans. Int J Syst Evol Microbiol 2009;59:118-124.

4. Bouvet, P. J., and P. A. Grimont. 1986. Taxonomy of the genus Acinetobacter with the recognition of Acinetobacter baumannii sp. nov., Acinetobacter haemolyticus sp. nov., Acinetobacter johnsoniisp. nov., and Acinetobacter junii sp. nov., and emended description of Acinetobacter calcoaceticus and Acinetobacter Iwoffii. Int. J. Syst. Bacteriol. 36:228-240.

5. Jones CL, Clancy M, Honnold C, et al. Fatal outbreak of an emerging clone of extensively drug-resistant Acinetobacter baumannii with enhanced virulence. Clin Infect Dis 2015, 61(2):145-54.

6. Choi WS, Kim SH, et al. Nosocomial Outbreak of Carbapenem - Resistant Acientobacter baumannii in Intensive Care Units and Successful Outbreak Control Program. Journal of Korean Medical Science 2010;7:999-1004

7. Wright MS, lovleva A, Jacobs MR, et al. Genome dynamics of multidrug-resistant
Acinetobacter baumannii during infection and treatment. Genome Med 2016;8(1):26.

8. Asif M, Alvi IA, Rehman SU. Insight to Acinetobacter baumannii:pathogenesis, global resistance, mechanisms of resistance, treatment options, and alternative modalities

9. Peleg AY, Seifert H, Paterson D. Acinetobacter baumannii: Emergence of a Successful Pathogen. Clin Microbiol Rev 2008;21(3):538-582

10. Nemec A, De Baere T, Tjernberg I, Vaneechoutte $M$, van der Reijden TJ, Dijkshoorn L. Acinetobacter ursingii sp. nov. and Acinetobacter schindleri sp. nov., isolated from human clinical specimens. Int J Syst Evol Microbiol 2001;51(Pt 5):1891-9.

11. Nemec A, Dijkshoorn L, Cleenwerck I, De Baere $\mathrm{T}$, et al. Acinetobacter parvus sp. Nov., a small-colony-forming species isolated from human clinical specimens. Int $\mathrm{J}$ Syst Evol Microbiol 2003; 53(Pt4):1563-7.

12. Carr, E. L., P. Kampfer, B. K. Patel, V. Gurtler, and R. J. Seviour. Seven novel species of Acinetobacter isolated from activated sludge. Int. J. Syst. Evol. Microbiol 2003. 53:953-963.

13. Tjernberg, I., and J. Ursing. Clinical strains of Acinetobacter classified by DNA-DNA hybridization. APMIS 1989;97:595-605.

14. Garner-Smidt P, Tjernberg I, Ursing J. Reliability of phenotypic tests for identification of Acinetobacter species. J Clin Microbiol 1991; 29:277-282.

15. Murray PR, Baron EJ, Pfaller MA, Tenover FC, Yolken RH. Manual of Clinical Microbiology, 6th edition. Washington DC: American Society of Microbiology, 1995:190-208.

16. Hartstein AI, Morthland VH, Rourke JW Jr, Freeman J, Garber S, Sykes R, Rashad $A L$. Plasmid DNA fingerprinting of Acinetobacter calcoaceticus subspecies anitratus from intubated and mechanically ventilated patients. Infect Control Hosp Epidemiol 1990;11(10):531-8.

17. Bou G, Cerveró G, Domínguez MA, Quereda C, Martínez-Beltrán J. PCR-based DNA fingerprinting (REP-PCR, AP-PCR) and pulsed-field gel electrophoresis characterization of a nosocomial outbreak caused by imipenem- and meropenem-resistant Acinetobacter baumannii. Clin Microbiol Infect. 2000;6(12):635-43.

18. Seifert H, Dolzani L, Bressan R, van der Reijden T, van Strijen B, Stefanik D, Heersma H, Dijkshoorn L. Standardization and interlaboratory reproducibility assessment of pulsed-field gel electrophoresis-generat- 
ed fingerprints of Acinetobacter baumannii. J Clin Microbiol. 2005;; 43(9):4328-35.

19. Janssen P, Coopman R, Huys G, Swings J, Bleeker M, Vos P, Zabeau M, Kersters K. Evaluation of the DNA fingerprinting method AFLP as an new tool in bacterial taxonomy. Microbiology. 1996; 142:1881-93.

20. Al Sehlawi ZS, Almohana AM, Thahab AA. Isolation and Identification of Acinetobacter baumannii Clinical Isolates using Novel Methods. Journal of Babylon University 2014; 22:2-11.

21. Lugtenberg B, van Alphen L. Molecular architecture and functioning of the outer membrane of Escherichia coli and other gram-negative bacteria. Biochim Biophys Acta 1983. 737:51-115

22. Pantophlet R, Brade L, Brade H. Identification of Acinetobacter baumannii Strains with Monoclonal Antibodies against the $\mathrm{O}$ Antigens of Their Lipopolysaccharides. Clinical and Diagnostic Laboratory Immunology 1999;6:323-329.

23. Haseley SR, Holst O, Brade H. Structural and serological characterization of the O-antigenic polysaccharide of the lipopolysaccharide from Acinetobacter haemolyticus strain ATCC 17906. Eur J Biochem 1997;244:761-766.

24. Haseley SR, Holst O, Brade H. Structural studies of the O-antigen isolated from the phenol-soluble lipopolysaccharide of Acinetobacter baumannii (DNA group 2) starin 9. Eur J Biochem 1998;251:189-194.

25. Baig A, Cabral TM, Corbett CR. Development and characterization of monoclonal antibodies for rapid detection of Acinetobacter baumannii. Monoclon Antib Immunodiagn Immunother 2014;33(4):291-8.

26. Thoma C, Bacchi M, Bedenic B, Cornaglia G, Mazzariol A. Evaluation of molecular and biochemical methods to detect carbapenemases-producing Acinetobacter baumannii. 2016

27. Doi Y, Murray GL, Peleg AY. Acinetobacter baumannii: Evolution of Antimicrobial Resistance-Treatment Options. Semin Respir Crit Care Med 2015;36:85-98.

28. Shimose LA, Masuda E, Sfeir M, et al. Carbapenem-resistant Acinetobacter baumannii: concomitant contamination of air and environmental surfaces. Infect Control Hosp Epidemiol 2016;37(7):777-781.

29. Tudor CA, Boros C, Petre R, et al. Raspunsul imun in pneumonia cu Acinetobacter baumannii. Revista romana de boli infectioase 2016;19(1):16-21.

30. Greene C, Vadlamudi G, Newton D, Foxman
$B, X i C$. The influence of biofilm formation and multidrug resistance on environmental survival of clinical and environmental isolates of Acinetobacter baumannii. Am J Infect Control 2016;44(5):e65-71.

31. Moreira Silva G, Morais L, Marques L, Senra V. Acinetobacter community-acquired pneumonia in a healthy child. Rev Port Pneumol 2012;18(2):96-8.

32. Arodino I, Zangirolami F, lemmi D, Lanzoni $\mathrm{M}$, Cargnelutti $\mathrm{M}$, et al. Risk factors and epidemiology of Acinetobacter baumannii infections in a university hospital in Northern Italy: A case-control study.

33. Zarrilli R. Acinetobacter baumannii virulence determinants involved in biofilm growth and adherence to host epithelial cells. Virulence 2016;1-2.

34. Denton M, Wilcox MH, Parnell P, et al. Role of environmental cleaning in controlling an outbreak of Acinetobacter baumannii on a neurosurgical intensive care unit. Intensive Crit Care Nurs 2005;21(2)94-98.

35. Orsi GB, Franchi C, Giordano A, et al. Multidrug-resistant Acinetobacter baumannii outbreak in an intensive care unit. J Chemother 2008;20(2):219-228.

36. Gaynes R, Edwards JR. Overview of nosocomial infections caused by Gram-negative bacilli. Clin. Infect Dis 2005;41:848-854.

37. Qiu H, Kuo Lee R, Harris G, van Rooijen $\mathrm{N}$, et al. Role of Macrophages in Early Host Resistance to Respiratory Acinetobacter baumannii Infection. PLoS One 2012;7(6): e40019.

38. Valencia R, Arroyo LA, Conde M, et al. Nosocomial outbreak of infection with pan-drug-resistant Acinetobacter baumannii in a tertiary care university hospital. Infect Control Hosp Epidemiol. 2009;30(3):25763.

39. Pourhajibagher M, Hashemi FB, Pourakbari B, Aziemzadeh M, Bahador A. Antimicrobial Resistance of Acinetobacter baumannii to Imipenem in Iran: A Systematic Review and Meta-Analysis. Open Microbiol J 2016; 10():32-42.

40. Marvig R L, Johansen HK, Molin S, Jelsbak L.Genome analysis of a transmissible lineage of Pseudomonas aeruginosa reveals pathoadaptive mutations and distinct evolutionary paths of hypermutators. PLoS Genet. 2013

41. Hua X, Zhou Z, Yang Q, et al. Evolution of Acinetobacter baumannii In Vivo: International Clone II, More Resistance to Ceftazidime, Mutation in ptk. Front Microbiol 2017;8:1256. 
42. Bergogne-Bérézin E, Towner KJ. Acinetobacter spp. as nosocomial pathogens: microbiological, clinical, and epidemiological features. Clin Microbiol Rev 1996;9(2):14865.

43. Da Silva G, Dijkshoorn L, van der Reijden $\mathrm{T}$, van Strijen B, Duarte A. Identification of widespread, closely related Acinetobacter baumannii isolates in Portugal as a subgroup of European clone II. Clin Microbiol Infect. 2007 Feb; 13(2):190-195.

44. Turton JF, Kaufmann ME, Warner M, Coelho J, Dijkshoorn L, van der Reijden T, Pitt TL. A prevalent, multiresistant clone of Acinetobacter baumannii in Southeast England. J Hosp Infect. 2004;58(3):170-9.

45. 382. Naas, T., B. Coignard, A. Carbonne, K. Blanckaert, O. Bajolet, C. Bernet, X. Verdeil, P. Astagneau, J. C. Desenclos, and P. Nordmann. 2006. VEB-1 extended-spectrum beta-lactamase-producing Acinetobacter baumannii, France. Emerg. Infect. Dis.12:1214-1222.

46. van Dessel H, Dijkshoorn L, van der Reijden T, Bakker N, Paauw A, van den Broek $P$, Verhoef J, Brisse S. Identification of a new geographically widespread multiresistant Acinetobacter baumannii clone from European hospitals. Res Microbiol. 2004;155(2):105-12.

47. Hujer KM, Hamza NS, Hujer AM, et al. Identification of a new allelic variant of the Acinetobacter baumannii cephalosporinase, ADC-7 $\beta$-lactamase: defining a unique family of class $C$ enzymes. Antimicrob Agents Chemother. 2005;49(7):29412948.

48. Héritier C, Poirel L, Nordmann P. Cephalosporinase over-expression resulting from insertion of ISAba1 in Acinetobacter baumannii. Clin Microbiol Infect. 2006 Feb; 12(2):123-30.

49. Adams-Haduch JM, Paterson DL, Sidjabat $\mathrm{HE}$, et al. Genetic basis of multidrug resistance in Acinetobacter baumannii clinical isolates at a tertiary medical center in Pennsylvania. Antimicrob Agents Chemother. 2008;52(11):3837-3843.
50. Figueiredo S, Poirel L, Papa A, Koulourida $\mathrm{V}$, Nordmann P. Over-expression of the naturally occurring blaOXA-51 gene in Acinetobacter baumannii mediated by novel insertion sequence ISAba9. Antimicrob Agents Chemother. 2009;53(9):4045-4047.

51. Urban C, Go E, Mariano N, Rahal JJ. Interaction of sulbactam, clavulanic acid and tazobactam with penicillin-binding proteins of imipenem-resistant and -susceptible Acinetobacter baumannii. FEMS Microbiol Lett. 1995;125:193-198.

52. Shaw KJ, Rather PN, Hare RS, Miller GH. Molecular genetics of aminoglycoside resistance genes and familial relationships of the aminoglycoside-modifying enzymes. Microbiol Rev. 1993;57(1):138-163.

53. Liou GF, Yoshizawa S, Courvalin P, Galimand $\mathrm{M}$. Aminoglycoside resistance by Ar$\mathrm{mA}-\mathrm{mediated}$ ribosomal $16 \mathrm{~S}$ methylation in human bacterial pathogens. J Mol Biol. 2006;359(2):358-364.

54. Adams-Haduch JM, Paterson DL, Sidjabat $\mathrm{HE}$, et al. Genetic basis of multidrug resistance in Acinetobacter baumannii clinical isolates at a tertiary medical center in Pennsylvania. Antimicrob Agents Chemother. 2008;52(11):3837-3843.

55. Coyne S, Rosenfeld N, Lambert T, Courvalin $P$, Périchon $B$. Over-expression of resistance-nodulation-cell division pump AdeFGH confers multidrug resistance in Acinetobacter baumannii. Anti-microb Agents Chemother. 2010;54(10):4389_ 4393.

56. Beceiro A, Llobet E, Aranda J, et al. Phosphoethanolamine modification of lipid $A$ in colistin-resistant variants of Acinetobacter baumannii mediated by the pmrAB two-component regulatory system. Antimicrob Agents Chemother. 2011;55(7):3370_ 3379.

57. Moffatt JH, Harper M, Harrison $P$, et al. Colistin resistance in Acinetobacter baumannii is mediated by complete loss of lipopolysaccharide production. Antimicrob Agents Chemother. 2010;54(12):49714977. 